\title{
Ideal Drug for Frug Induced Sleep Endoscopy, Protocol Vs Dexmedetomidine- Systematic Review
}

\author{
Abdul Wadood Mohammed* \\ Department of ENT, King Faisal University, Saudi Arabia
}

Submission: June 9, 2016; Published: July 14, 2016

*Corresponding author: Abdul Wadood Mohammed, Department of ENT, College of Medicine, King Faisal University, Al Hassa, Saudi Arabia, Tel: 00966504673230; Email: wadood_abd@yahoo.co.in; wadood83@yahoo.com

\begin{abstract}
Drug induced sleep endoscopy is the most important investigation before surgical planning of obstructive sleep apnea patient. It is performed by artificially inducing sleep in a patient and performing nasal endoscopy in order to identify the site of upper airway obstruction. The most appropriate anesthetic agent would be that which induces sleep without any respiratory inhibition. Propofol has been widely used to perform drug induced sleep endoscopy. However, Dexmedetomidine is a newer drug which has shown to have many advantages making it suitable to drug induced nasal endoscopy. The present study is a systematic review of English literature which compares the utility of Propofol and dexmedetomidine in drug induced sleep endoscopy.

Keywords: Drugs induced sleep; Obstructive sleep apnea; Sleep endoscopy; Pharyngeal collapse

Abbreviations: OSAS: Obstructive Sleep Apnea Syndrome; SNE: Sleep Endoscopy, SNE: Sleep Naso Endoscopy; DISE: Drug-Induced Sleep Endoscopy; OSA: Obstructive Sleep Apnea
\end{abstract}

\section{Introduction}

Obstructive sleep apnea syndrome (OSAS) is a syndrome of intermittent dynamic upper airway obstruction occurring during sleep. It is associated with chronic hypoxia leading to systemic and pulmonary hypertension and many other co morbidities like the stroke. The mainstay of OSAS management is the identification of the site of obstruction causing the apneic or hypopnea episodes. Drug induced sleep endoscopy is the method of pharmacological induction of sleep in a patient and simultaneously performing an endoscopy to visualize the upper airway [1]. Many anesthetic agents have been used to pharmacologically induced sleep. An ideal agent would be that which would provide the best upper airway paralysis with least interference in the respiratory system. Midazolam, propofol, and dexmedetomidine are commonly used for drug induced sleep endoscopy. This study tries to review the research published in English literature regarding the same to determine the ideal drug available for sleep endoscopy.

History of sleep endoscopy: (Due to the difficulty in establishing the site of obstruction in the conscious patient who carries a diagnosis of OSA, the diagnosis and treatment of OSA is a complex and multidimensional issue (Croft and Pringle [2]).

Importance: (Sleep endoscopy, also known as sleep nasoendoscopy (SNE) or drug-induced sleep endoscopy (DISE), is a powerful tool for studying the dynamic airway in a sleeping patient with obstructive sleep apnea (OSA).

\section{Material and Methods}

A systematic literature search in the PubMed was performed using the search terms drug induced sleep endoscopy and dexmedetomidine (Figure 1). The review excluded case reports, an article in the language other than English and off-topic articles.

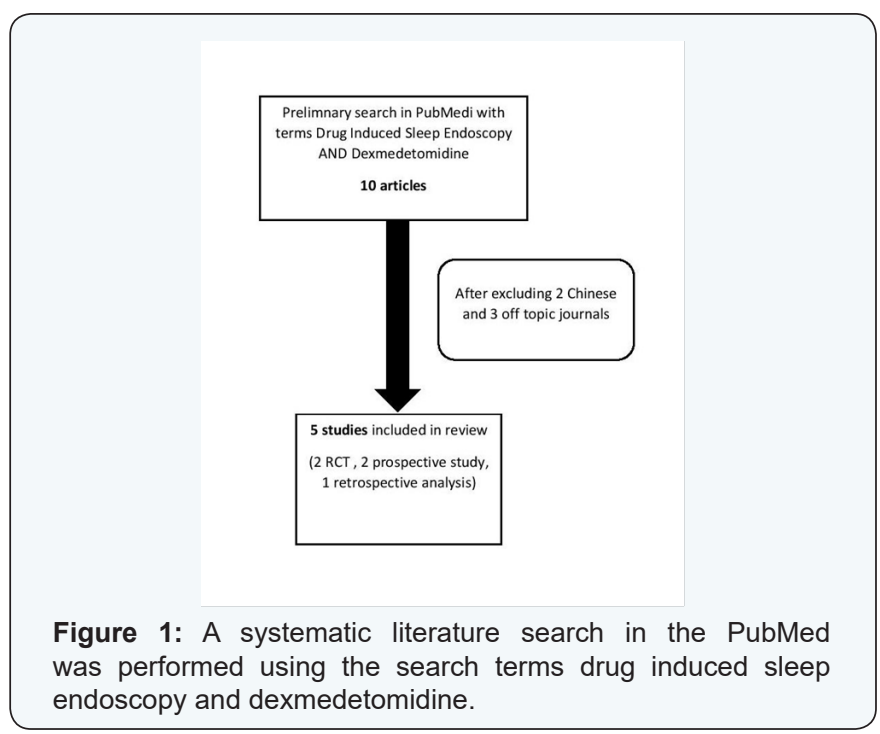




\section{Global Journal of Otolaryngology}

\section{Results}

The initial search in the PubMed revealed 10 articles. After excluding two Chinese articles and 3 articles which were off topic, 5 articles were included in the study which included two randomized controlled trials, one prospective observational study, one case series and one retrospective analysis (Table 1).

Table 1: Studies included in review.

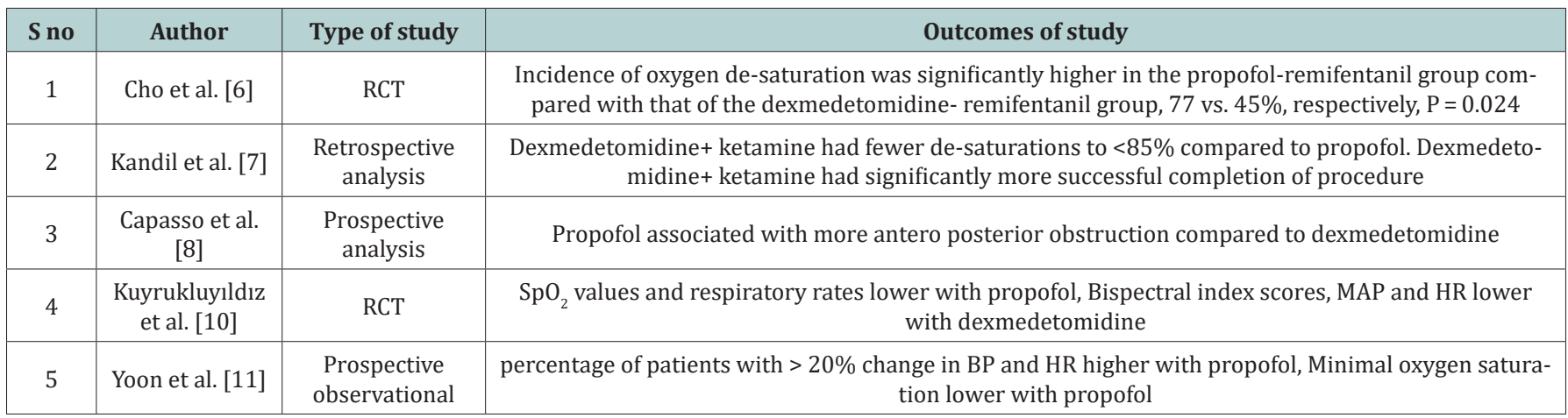

RCT: Randomized Controlled Trial; $\mathrm{SpO}_{2}$ : Oxygen Saturation; MAP: Mean Arterial Pressure; HR: Heart Rate; BP: Blood Pressure

\section{Discussion}

Drug induced sleep endoscopy is the first line investigation for patients with obstructive sleep apnea. Pringle and Craft [2] first performed it and published the results in 1993. This would allow the surgeon to identify the site of dynamic obstruction and accordingly plan for the surgical procedure. Generally, OSAS patients are more prone to anesthesia related morbidities and mortalities. Deep sedation and general anesthesia are the two common methods performed to induce artificial sleep. General anesthesia is usually preferred over deep sedation owing to the safety concerns. However, providing general anesthesia eliminate the possibility of a day care procedure. Hence, these two factors have to be assessed according to the patient status and the best method should be opted for.

Table 2: The comparison of properties of propofol and dexmedetomidine.

\begin{tabular}{|c|c|}
\hline Propofol & Dexmedetomidine \\
\hline 1. Action on GABA receptor & \\
2. Rapid onset of action & 1. Action - Alpha 2 agonistic in brain and spinal cord \\
3. Recovery in $10 \mathrm{~min}$ & 2. Half life 2 hours \\
4. Metabolized rapidly -hepatic \& extra hepatic mechanism & 3. 94\% protein bound \\
5. Side effects- Hypotension, respiratory depression, synergistic cardio- & 4. Eliminated by kidney and liver \\
vascular suppression with opioids & 5. Half life 2 hours \\
3. 94\% protein bound & 6. Side effects- over sedation, delayed awakening and analgesia without respiratory depression \\
4. Eliminated by kidney and liver & \\
5. Sedation and analgesia without respiratory depression &
\end{tabular}

Propofol and benzodiazepines are the most commonly used agents for drug induced sleep endoscopy. They can be used either individually or in combination. Dexmedetomidine is a newer high selective $\alpha-2$ adrenergic receptor agonist with anxiolytic and analgesic effects. The comparison of properties of propofol and dexmedetomidine is given in (Table 2). It does not produce respiratory depression and hence has the advantage of being a drug for day care procedures [3]. There are limited studies comparing the properties of these agents used for drug induced sleep endoscopy. The ideal agent is the one, which produces sedation without respiratory depression. The sedation produced by dexmedetomidine is considered as near to natural sleep compared to propofol. Dexmedetomidine is also associated with lesser upper airway musculature relaxation compared to propofol [4]. Respiratory depression is evidenced by oxygen de-saturation of the patient. Some amount of oxygen de-saturation is inevitable during anesthesia.

Hence, a cutoff level of $4 \%$ or more below the basal saturation value is considered a significant level of de-saturation [5]. A randomized controlled trial by Cho et al. [6] showed that propofolremifentanil combination produced more de-saturation compared to dexmedetomidine- remifentanil. However, this is seen at the 
expense of inadequate sedation with dexmedetomidine noticed in almost $50 \%$ of the patients who required unconscious sedation. In a retrospective review by Kandil et al. [7], propofol either alone or along with sevoflurane was associated with greater oxygen desaturation.

Another study by Capasso et al. [8] found that the use of propofol was associated with greater incidence of complete anteroposterior tongue base collapse. This finding may be attributed to the increased collapsibility of the upper airway due to profound relaxation of genioglossus muscle [9]. In another randomized controlled trial by Kuyrukluyildiz et al. [10] found that propofol provided more hemodynamic stability and dexmedetomidine provided more respiratory stability. Since obstructive sleep apnoea patients are more prone to respiratory complication, dexmedetomidine would be a preferred agent for drug induced sleep endoscopy. However, in another prospective review by Yoon et al. [11] dexmedetomidine provided both cardiovascular and respiratory stability compared to propofol.

\section{Conclusion}

In conclusion, dexmedetomidine is a better anesthetic agent for drug induced sleep endoscopy compared to propofol as it has greater hemodynamic and respiratory stability.

\section{References}

1. Ulualp So, Szmuk P (2013) Drug-induced sleep endoscopy for upper airway evaluation in children with obstructive sleep apnea. Laryngoscope 123(1): 292-297.

2. Pringle MB, Croft CB (1993) A grading system for patients with obstructive sleep apnoea-based on sleep nasendoscopy. ClinOtolaryngol Allied Sci 18(6): 480-484.
3. Hall JE, Uhrich TD, Barney JA, Arain SR, Ebert TJ (2000) Sedative, amnesic, and analgesic properties of small-dose dexmedetomidine infusions. Anesth Analg 90(3): 699-705.

4. Arain SR, Ebert TJ (2002) The efficacy, side effects, and recovery characteristics of dexmedetomidine versus propofol when used for intraoperative sedation. Anesth Analg 95(2): 461-466.

5. Rabelo FA, Braga A, Kupper DS, De Oliveira JA, Lopes FM, et al. (2010) Propofol-induced sleep: polysomnographic evaluation of patients with obstructive sleep apnea and controls. Otolaryngol Head Neck Surg 142(2): 218-224.

6. Cho JS, Soh S, Kim EJ, Cho HJ, Shin S, et al. (2015) Comparison of three sedation regimens for drug-induced sleep endoscopy. Sleep Breath 19(2): 711-717.

7. Kandil A, Subramanyam R, Hossain MM, Ishman S, Shott S, et al. (2016) Comparison of the combination of dexmedetomidine and ketamine to propofol or propofol/sevoflurane for drug-induced sleep endoscopy in children. Paediatr Anaesth 26(7): 742-751.

8. Capasso R, Rosa T, Tsou DY, Nekhendzy V, Drover D, et al. (2016) Variable Findings for Drug Induced Sleep Endoscopy in Obstructive Sleep Apnea with Propofol versus Dexmedetomidine. Otolaryngol Head Neck Surg 154(4): 765-770.

9. Eastwood PR, Platt PR, Shepherd K, Maddison K, Hillman DR (2005) Collapsibility of the upper airway at different concentrations of propofol anesthesia. Anesthesiology 103(3): 470-477.

10. Kuyrukluyıldız U, Binici O, Onk D, AyhanCelik S, Torun MT, et al. (2015) Comparison of dexmedetomidine and propofol used for drug induced sleep endoscopy in patients with obstructive sleep apnea syndrome. Int J ClinExp Med 8(4): 5691-5698.

11. Yoon BW, Hong JM, Hong SL, Koo SK, Roh HJ, et al. (2016) A comparison of dexmedetomidine versus propofol during drug induced sleep endoscopy in sleep apnea patients. Laryngoscope 126(3): 763-767. 